\title{
Regulation of the pulmonary circulation
}

\author{
G. de J. Lee \\ From the Cardiac Department, Radcliffe Infirmary, Oxford
}

Factors regulating pressure and flow in the lungs are reviewed with particular emphasis on their role in regulating blood flow velocity and distribution within the lung capillaries. The behaviour of the pulmonary arterial system, alveolar capillaries, and pulmonary venous system are considered individually. The effect of heart disease on lung capillary blood flow is examined.

The behaviour of the lung circulation has inspired intense study during the years since the International Society of Cardiology last met in 1966. This is partly owing to the fact that techniques are now available for use in man which give information with a precision previously available only to the investigator in the experimental laboratory. Thus contributions which clinicians have made to the better understanding of the physiological relationships governing both ventilation and blood flow in the lungs have also led to greater accuracy in diagnosis and a more rational approach to treatment.

The prime function of the lungs is to accomplish efficient gas exchange between the blood flowing through the pulmonary capillaries and the air ventilating their related alveoli. Normally this process takes place over an enormous range of activity with the cardiac output approaching $301 . / \mathrm{min}$. in extreme exercise. Yet the lung capillary blood volume probably never exceeds $200 \mathrm{ml}$. (Roughton and Forster, 1957; Bates et al., 1960; Weibel, I963), and blood flow is accomplished in such a manner that the intravascular pressures within the capillaries rarely exceed the plasma osmotic filtration pressure, so that the Starling relation between the vascular and extravascular compartments of the lungs is maintained and pulmonary oedema is avoided.

In disease of the lungs, adaptive mechanisms operate which redistribute both blood flow and gas delivery away from diseased areas to the more normal parts of the lung; while in heart disease, particularly those conditions leading to increased impedance to venous outflow from the lungs into the left side of the heart, mechanisms again develop which partly protect the lung capillaries from experiencing the high vascular pressures present in the larger blood vessels as a consequence of the heart disease. This assists in preserving the alveolar capillary system as a gas exchanging area rather than converting it to a plasma filtration system, manifest clinically by pulmonary oedema. The activity of the lung lymphatic system in draining the extravascular spaces of the lung also assists in preserving the gaseous environment of the alveoli.

The peripheral lung blood vessels also act as a sieve and as a lytic environment capable of removing particulate material such as platelet aggregates, etc., delivered to it from the systemic venous system, which would be dangerous to the organism if allowed to continue to circulate to the systemic arterial system.

Finally, the systemic circulation contributes to the overall regulation of blood flow in the lungs via the bronchial circulation. In health the bronchial circulation probably contributes less than 12 per cent of the total lung blood flow, but in lung disease bronchopulmonary anastomoses play an increasingly important part in local perfusion of diseased areas (Cudkowicz, 1968).

This brief description of functions ignores the more occult participation of the lung circulation in the organ's metabolic and humoral activity with regard to such substances as histamine, 5-hydroxy tryptamine, and other circulating catecholamines, lipid and cholesterol metabolism, etc. (Heinemann and Fishman, 1969).

This brief review of functions involving the lung circulation indicates its complexities. Not unnaturally, the regulation of the pulmonary circulation depends on an equally complex variety of factors whose relationships to one another result in overall homoeostasis.

Fig. I lists these interrelationships in diagramatic form. The 'active' vasomotor effects 


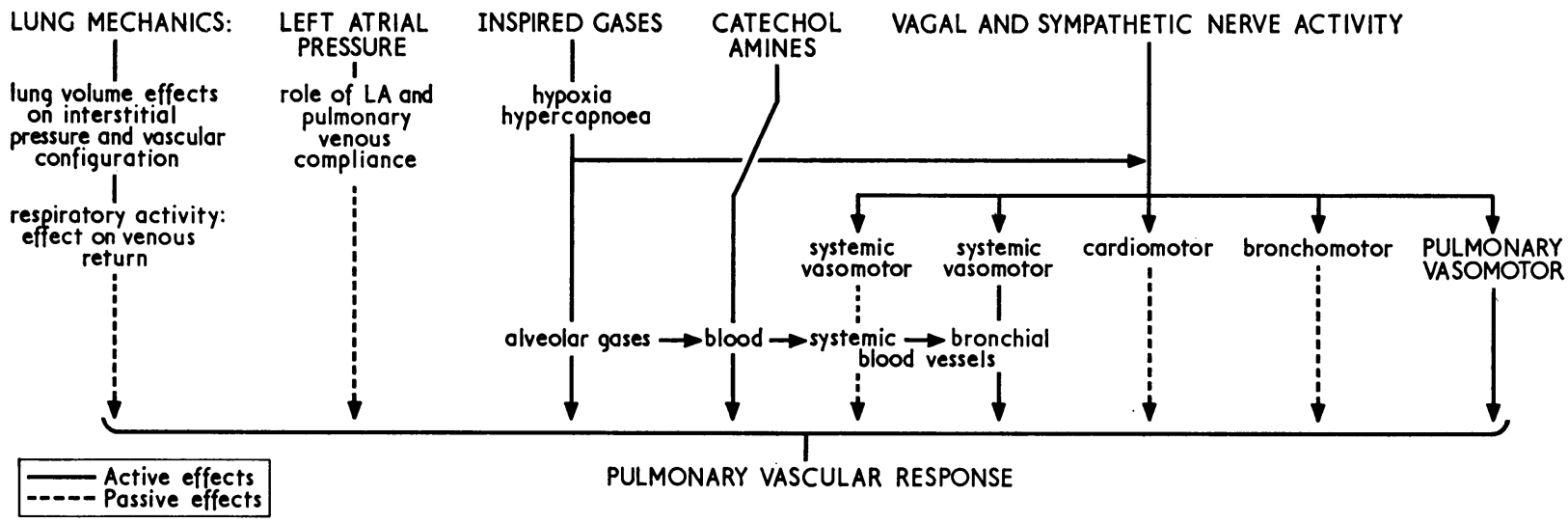

FIG. I Active and passive factors affecting the pulmonary vascular bed.

referred to in the figure indicate responses which occur in the pulmonary blood vessels manifest by contraction or relaxation of their own walls due to humoral, chemical, or reflex stimuli; while ' passive' effects refer to changes imposed upon the pulmonary blood vessels from mechanical events within the thorax and lungs, or secondary to circulatory events taking place elsewhere in the body.

A detailed analysis of the regulatory functions of each of the activities listed in Fig. $I$ is beyond the scope of this presentation. I therefore intend to confine myself to an examination of what I believe to be the most important factors responsible for regulating blood flow within the lung capillaries themselves: for the ultimate test of the lung's efficiency must surely be its capability to maintain adequate conditions for gas exchange over the widest possible range of physiological activity and pathological insult.

\section{Nature of lung capillary blood flow}

The lung circulation in normal man has a low peripheral resistance and a low inherent reflex vasomotor activity (Lee, Matthews, and Sharpey-Schafer, 1954). Thus one might expect that the pulsatile ejection of blood flow from the right ventricle might continue to be transmitted through the pulmonary arterial system so that capillary blood flow also remained pulsatile. Lee and DuBois (1955) proved this to be the case by measuring the lung capillary blood flow instantaneously throughout the cardiac cycle by continuously recording the rate of nitrous oxide uptake from the lungs using the body-plethysmograph method. They found that the lung capillary blood flow was highly pulsatile with each cardiac cycle. These events were so rapid that some passive rather than reflex vascular adjustment could be presumed to exist for regulating flow and pressure. It could also be inferred from the fact that the fully distended lung capillary system has a blood volume almost identical to the stroke volume of the heart, while many previous workers had demonstrated that pulmonary vascular resistance always decreased when blood flow through the lungs increased during exercise.

\section{Blood flow distribution in lungs: ventila- tion-perfusion relationships}

The hydrostatic mechanism which operates to reduce lung vascular resistance as blood flow increases also serves to regulate distribution of blood flow within the lungs. Its existence was first discovered by Banister and Torrance in I960 in isolated perfused lungs. They found that the interrelation between alveolar pressure, tending to close lung capillaries, and the arterial and venous pressures, tending to open them, regulated blood flow. The alveolar capillary systems were acting effectively as Starling resistors.

In the same year West and Dollery (1960), using radioactive gases, had found in man that blood flow at the lung bases was some eight times greater than at the apices, while the distribution of ventilation was also somewhat greater to the lower zones than it was to the upper zones of the lungs. Intense study of lung ventilation-perfusion distribution followed, led particularly by Permutt, Bromberger-Barmea, and Bane (1962) in the United States, and West, Dollery, and Naimark (1964) in Britain.

In the vertically suspended isolated, ventilated, and perfused lung it was found that no capillary blood flow took place at hydrostatic levels above which alveolar pressure exceeded both the pulmonary arterial and venous pres- 
sures (West: Zone I). Flow began at a level down the lung where pulmonary arterial pressure exceeded alveolar pressure (West: Zone 2). It continued to rise in a manner determined by the difference between pulmonary arterial and alveolar pressures until a level was reached where the pulmonary venous pressure also exceeded the alveolar pressure. Over the remainder of the dependent lung blood flow was then governed by the pulmonary arterialvenous pressure difference (West: Zone 3 ), for in this zone both these pressures exceeded the alveolar pressure and the capillary systems were thus permanently open.

Hughes et al. (1968) subsequently extended this work to discover the effects of changes in lung volume on the regional distribution of blood flow and found that vessels not directly exposed to alveolar pressure affected vascular resistance in the lowermost parts of the lung in an unexpected way. These so-called extraalveolar vessels of the lung are held open by the degree of expansion of the lung (Rosenzweig, Hughes, and Glazier, 1970). However, the base of the upright lung is relatively poorly expanded, so that the extra-alveolar vessels significantly reduce blood flow in the lowermost zones. Hughes and his colleagues were able to show a fourth zone at the extreme base of the lung, where regional blood flow began to fall again in spite of a continuing rise in intravascular hydrostatic pressure.

In the past, Howell et al. (I96I) and Permutt (1965) had produced evidence that lung inflation increased the capacity of those larger lung vessels outside the influence of alveolar pressure. These vessels were in effect sucked upon by the negative interstitial pressure about them. Mead, Takishima, and Leith (1967) suggested that the negative distending pressure transmitted to these vessels depended on the extent to which they failed to take part in the expansion process of the lung in inspiration. The interstitial tissues in these areas would then tend to have disproportionately higher negative pressures about them, so that interstitial fluid accumulation would tend to be greater at the base of the lung than in the upper zones, thus contributing to the increased vascular resistance to blood flow found in Zone 4 by Hughes and his colleagues. This has important clinical relevance, for it seems most probable that the increased pulmonary venous pressure which results from left-sided heart disease will increase fluid transudation at the lung bases long before overt pulmonary oedema is recognized. This will further increase impedance to blood flow through the lower zones of the lungs and will result in the preferential redistribution of blood flow to the upper zones of the lungs so commonly seen in mitral stenosis.

These hydrostatic relationships describe blood flow distribution in the lungs under steady flow conditions. They plainly depend on the role of alveolar capillary systems acting as parallel resistors stacked vertically, and indicate how vascular pressure adjustments as well as zonal distribution of flow could be achieved under real life conditions of pulsatile blood flow. Thus at peak capillary flow rate the pulmonary arterial pressure will momentarily exceed the alveolar gas pressures in all areas of the lungs, so that all lung capillary systems will open to accommodate blood flow. During diastole, alveolar capillary systems will cease to conduct the flow as the pulmonary arterial pressure falls in their zone. This will begin in the uppermost zones of the lung and extend downwards as arterial pressure falls during diastole. Thus regional lung perfusion will vary with time throughout the cardiac cycle in a tidal manner, regulated by gravity and becoming most uniform momentarily during the peak of systole, or when exercise increases the rate of blood flow so that the pulmonary arterial pressure is permanently elevated above alveolar pressure.

This tidal concept for accommodating pulsatile capillary blood flow implies a fluctuating capillary volume participating in gas diffusion at low levels of cardiac output, while at high output levels, when all capillaries are permanently recruited, increased gas diffusion will be achieved by pulsatile changes in blood velocity through each capillary system.

Du Bois and his colleagues (Menkes et al., 1970) have now demonstrated that the lung capillary volume does indeed fluctuate throughout the cardiac cycle in normal erect subjects at rest. They measured this by an ingenious modification of the single breath $\mathrm{D}_{\mathrm{co}}$ method, using a water-filled body plethysmograph. They showed that the CO uptake from five normal subjects indicated large capillary volume changes during each cardiac cycle. The greatest volume increase during the cycle coincided with peak capillary blood flow rates measured by the $\mathrm{N}_{2} \mathrm{O}$ uptake method in the same subject.

Lung ventilation is also found to be inhomogeneous, for it also increases down the lung but less rapidly than does blood flow under normal conditions. Milic-Emili and his colleagues (1966) have shown that subjects inspiring after full exhalation filled the upper zones of their lungs first. When a certain lung volume was reached the lower zones began to fill better than the upper zones. This is due to the way the lungs are supported in the thorax. 
It appears that the hilum takes very little of the weight of the lung, which is largely supported by the pleural surfaces consequent upon the negative intrathoracic pressure. Because of gravity, the lower regions are relatively compressed by the weight of the lung above them, and the upper zones are relatively overexpanded by the weight of the lung below. These differences can be shown by the fact that the intrapleural pressure is less negative at the bottom of the upright lung than at the top of it. A further consequence of this gravitational effect is the fact that the lung alveoli decrease in size from apex to base in the upright lung (Glazier et al., 1966, 1968).

\section{Haemodynamic factors affecting pulsa- tile capillary blood flow}

Having briefly reviewed the mechanical effects that the physical environment within the thorax imposes upon the lung circulation and the distribution of pulmonary capillary blood flow in particular, I now wish to examine the effect that changes in the intrinsic behaviour of the pulmonary circulation itself may have on lung capillary blood flow. The pulsatile nature of lung capillary blood flow depends upon the instantaneous pressure events taking place simultaneously at the arteriolar and venular ends of the capillary system. These in turn depend upon simultaneous events in the right ventricle and left atrium of the heart, modified by the physical characteristics of the pulmonary arterial and venous systems linking these chambers to the capillary system.

The nitrous oxide/body plethysmograph method for measuring the lung capillary blood flow, combined with simultaneous measurements of vascular pressures, has provided special opportunity to examine the effects of changes in the pulmonary arterial and venous system upon lung capillary blood flow pulsatility.

We have developed a recording system for use with the whole body plethysmograph which allows us to measure the rate of $\mathrm{N}_{2} \mathrm{O}$ uptake from the lungs with a frequency response that is flat to 15 cycles per second (Bosman et al., 1964; Karatzas, Lee, and Stott, 1967), so that we are now able to compare dynamic flow events with the same accuracy as simultaneous pressure events within the lung circulation and submit both to Fourier analysis when required.

Fig. 2 shows an example of an $\mathrm{N}_{2} \mathrm{O}$ uptake record superimposed upon a previously obtained air control record from a normal subject lying in the body plethysmograph. The records are timed against the electrocardiograph and phonocardiogram in order to

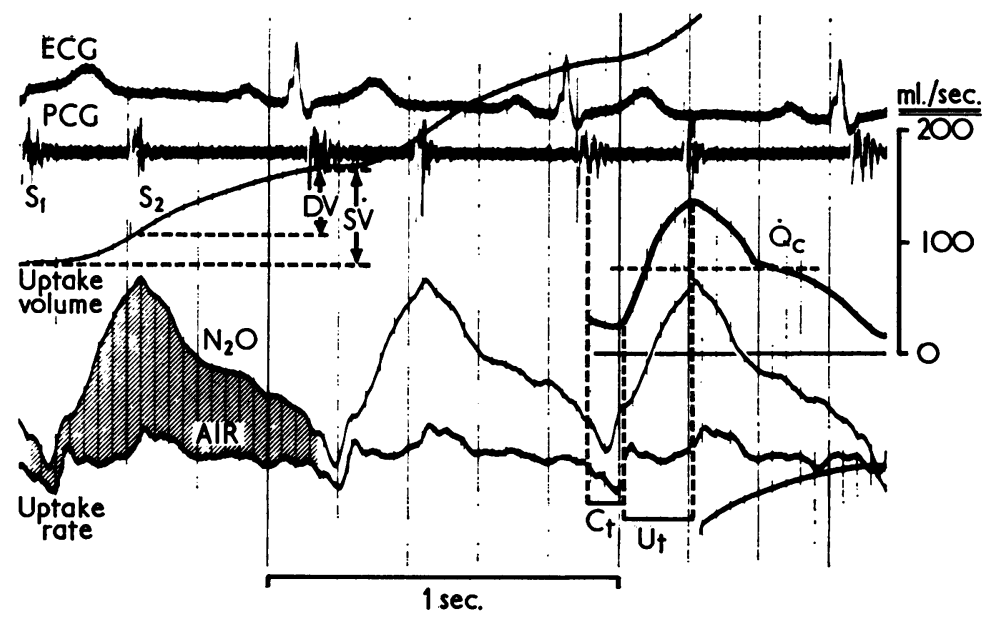

FIG. 2 Parts of an air control record (AIR) and $\mathrm{N}_{2} \mathrm{O}^{-}$uptake record $\left(\mathrm{N}_{2} \mathrm{O}\right)$ obtained with identical heart rates, superimposed upon one another.

The electrocardiogram and phonocardiogram

$(P C G)$ are displayed for timing purposes.

The shaded area on the left corresponds to the volume of $\mathrm{N}_{2} \mathrm{O}$ absorbed in one cardiac cycle; this is also given by the integrator $(S V)$.

$D V=\mathrm{N}_{2} \mathrm{O}$ uptake during diastole.

$C t=$ Flow conduction time.

$\mathrm{Ut}=\mathrm{N}_{2} \mathrm{O}$ upstroke time.

$\dot{Q}_{c}=$ Capillary flow rate calculated from $\mathrm{N}_{2} \mathrm{O}$-air records and redrawn.

time the opening and closing of the pulmonary valve $\left(S_{1}, S_{2}\right.$, respectively). The shaded area on the left of the figure indicates the volume of $\mathrm{N}_{2} \mathrm{O}$ taken up by the lungs in one cardiac cycle, which is also given by the integrator signal (SV). On the right of the figure is plotted the absolute capillary blood flow rate (Q) obtained from the shaded $\mathrm{N}_{2} \mathrm{O}$ curve. The average acceleration of blood flow in the lung capillaries can be measured from the upstroke time (Ut) of the $\mathrm{N}_{2} \mathrm{O}$ curve.

In addition, the percentage of the right ventricular stroke volume stored in the pulmonary arterial system during systole can be calculated. This pulmonary arterial systolic storage volume can be measured by subtracting the volume of blood flowing from the lung capillaries during diastole from the total stroke volume (stroke volume minus diastolic volume: $S V-D V)$.

The degree of pulmonary capillary flow pulsatility can be quantitated as a ratio between the peak flow rate to mean flow rate ( $\dot{\mathrm{Q}} \mathrm{max} / \overline{\mathrm{Q}} \mathrm{c}$ ). This is termed the pulsatility index.

The method also allows one to measure the 
time it takes the flow pulse to travel from the pulmonary valve to the lung capillaries. This is timed either from the first upstroke of the pulmonary artery pressure measured at cardiac catheterization to the time of beginning of $\mathrm{N}_{2} \mathrm{O}$ uptake in the body plethysmograph, or it may be timed from the first heart sound of the phonocardiogram $\left(S_{1}\right)$ to the foot of the capillary flow pulse measured by $\mathrm{N}_{2} \mathrm{O}$ uptake. This is termed the pulmonary arterial conduction time $(\mathrm{Ct})$. Our findings from normal subjects have shown that the lung capillary pulsatility index ( $\dot{Q} c \max / \bar{Q} c)$ has an approximate ratio of 2:I (Karatzas and Lee, 1966). This value agreed closely with similar studies reported by Wasserman, Butler, and Van Kessel (1966). The normal PA-Pc conduction time averaged $120 \pm 7.0 \mathrm{msec}$., while the systolic acceleration rate (Ut) was $8 \cdot 2 \pm \mathrm{I} \cdot 7$ $\mathrm{ml} . / \mathrm{sec} . / \mathrm{msec}$. (Karatzas, and Lee, 1969).

Of particular interest was the finding that the systolic storage volume of the pulmonary arterial system in normal resting subjects whose heart rate averaged $6 \mathrm{r} / \mathrm{min}$. averaged 66 per cent of the total stroke volume. When the heart rate was increased by administering atropine there was a highly significant fall in the storage capacity of the pulmonary arterial system, so that at an average heart rate of $\mathrm{IrO} / \mathrm{min}$. the storage volume had decreased to 56 per cent of the stroke volume (Karatzas et al., 1969). This finding is really an indication of the supply capacity of the pulmonary arterial system available for maintaining efficiency of gas exchange in the lung capillaries during exercise. Thus during exercise, when the heart rate increases, the diastolic period of the cardiac cycle shortens so that blood flow is presented to the lung capillaries for gas exchange in a more uniform manner with respect to time than under resting conditions. This, coupled with the more uniform spatial distribution of blood flow within the lungs during exercise, compared with the resting state, explains the facility with which the lung is able to increase its gas exchange capacity over a wide range of exercise.

Our early clinical comparisons of simultaneous pulmonary vascular pressure and pulmonary capillary flow measurements suggested that a clinical verification of the analogue studies by Wasserman et al. (1966) would be worth while. For in patients with various forms of heart disease leading to pulmonary hypertension, the capillary flow pulsatility index became attenuated when the pulmonary arterial resistance rose (Karatzas and Lee, 1966). We naturally hoped that simply measuring the pulsatility index by recording $\mathrm{N}_{2} \mathrm{O}$ uptake in the body plethysmo- graph would prove to be a useful noninvasive method for deriving pulmonary arterial resistance. Moreover, the shape of the normal capillary flow curve was so similar to the pulmonary artery - left atrial pressure difference, responsible for propelling blood through the lung capillary system, that we also hoped for useful diagnostic aid by comparing the $\mathrm{N}_{2} \mathrm{O}$ uptake curve with the $\mathrm{PA}$ - pulmonary wedge pressure differences, in order to obtain similar noninvasive evidence of instantaneous pressure events in these two sites by recording their effect on capillary blood flow. This we hoped would be particularly useful in differentiating stenosis from incompetence in patients with mixed mitral valve disease. Our hopes were too sanguine, as is shown by our results from 36 patients with heart disease leading to various combinations of pulmonary arterial and pulmonary venous hypertension (Karatzas and Lee, 1970). Fig. 3 shows the results we obtained. It can be seen that there was a negative correlation between the pulmonary capillary pulsatility index $(\dot{Q} c \max / \bar{Q} c)$ and each of the following: (I) mean pulmonary arterial pressure; (2) left atrial pressure, measured indirectly as the pulmonary arterial wedge pressure; and (3) the pulmonary arterial resistance.

These findings showed that patients with heart disease affecting the pulmonary circulation developed abnormal pulmonary capillary flow patterns, but that the degree and type of lung capillary flow abnormality could not be used alone to predict either the severity or the form of the pulmonary vascular disease present. For, though patients with raised pulmonary vascular resistance tended to have low pulsatility indices, so did patients with poor right ventricular ejection, low stroke volume, or tachycardia. Moreover, patients with high left atrial pressure but without severe pulmonary arterial hypertension also tended to have capillary flow pulses of diminished amplitude with an abnormal configuration, particularly those patients with predominant mitral incompetence. Thus an increase in pulmonary arterial resistance or an increased pulmonary venous input impedance, or a combination of the two, could produce attenuation of the pulmonary capillary flow pulse. Subsequently we have found a few examples of patients with high pulmonary arterial pressures in whom the capillary flow pulsatility index has remained remarkably normal. This reminded us of findings by Fishman and his colleagues (Morkin, Levine, and Fishman, 1964), who had studied the effects of induced pulmonary hypertension by hypoxia on pulmonary capillary flow pulsa- 
(a)

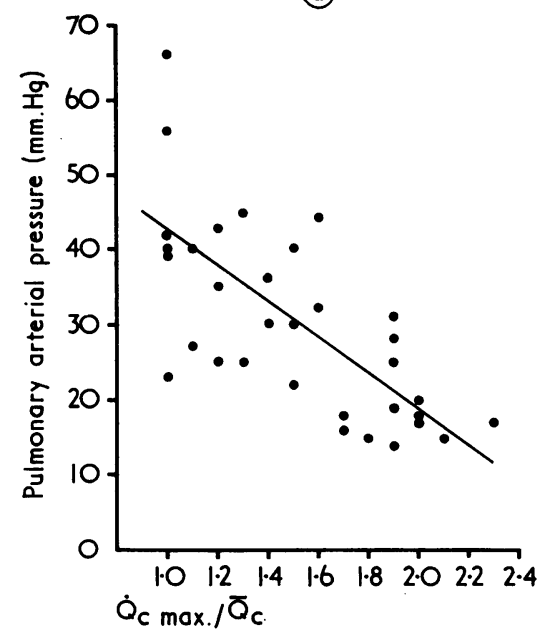

(b)

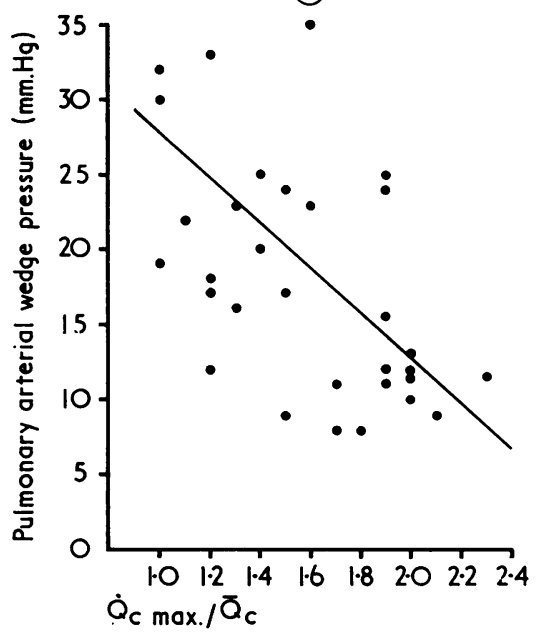

(c)

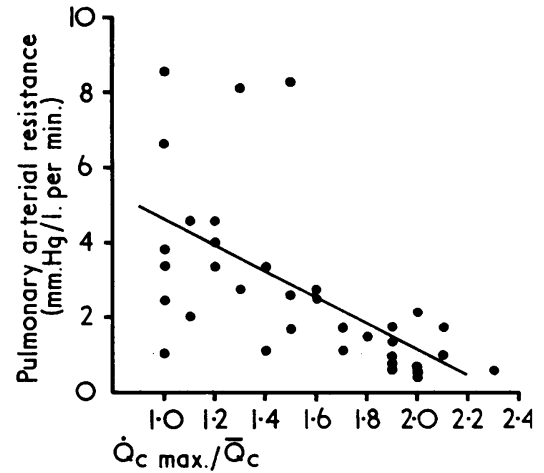

FIG. 3 Correlation between the ratio of peak to mean capillary flow rates and (a) mean pulmonary artery pressure, (b) mean pulmonary arterial wedge pressure, and (c) pulmonary arterial resistance in 36 patients with heart disease.

tility in the dog. They found that in spite of an obvious rise in pulmonary vascular resistance the pulsatility index remained unaltered with hypoxia.

We thought that the explanation of both this finding and our clinical finding that the pulsatility index sometimes remained normal at high pulmonary artery pressures could be due to a combined effect of a simultaneous reduction of pulmonary arterial distensibility or compliance $(\mathrm{C})$ occurring in conjunction with the increased resistance $(R)$ responsible for the elevated pressures in both instances. If (C) were to fall as (R) were rising, then the time constant of the system $(C \times R)$ would remain constant over a wide range of pulmonary arterial pressure, thus preserving an unaltered flow pulsatility in the capillaries.

We therefore tested this hypothesis in animals. The pulmonary capillary blood flow was measured in the body plethysmograph simultaneously with pulmonary arterial inflow using a cuff electromagnetic flowmeter, pulmonary artery pressure, left atrial pressure, and computed pulmonary arteriolar pressure in closed-chested atrially paced dogs. The left atrial pressure was below $5 \mathrm{~cm}$. of $\mathrm{H}_{2} \mathrm{O}$ in all instances. The effect of tachycardia, hypoxia $\left(10 \% \mathrm{O}_{2}\right)$, and serotonin infusion was studied. The pulmonary arterial volume was calculated from the ether circulation time from an injection site at the pulmonary valve to the lung capillaries in the body plethysmograph (Feisal, Soni, and DuBois, 1962; Sackner, Will, and DuBois, 1966). We next calculated the pulmonary arterial resistance conventionally and obtained pulmonary arterial compliance (C) by dividing the systolic storage volume (PA inflow - Pc outflow during systole $=\Delta \mathrm{V}$ ) by the mean PA systolic distending pressure $((\mathbf{P A p}+\mathbf{P}$ arteriolar $\mathrm{p}) / \mathbf{2}=\Delta \mathbf{P})$.

The pulmonary arterial distensibility (D) was calculated as the ratio of the lumped compliance (C) to the mean pulmonary arterial volume (Vo) as determined by the ether plethysmograph method and expressed as a percentage volume change per unit pressure thus:

$$
\mathrm{D}=\frac{\Delta \mathrm{V}}{\Delta \mathrm{P} \times \mathrm{Vo}} \times 100
$$

where $\Delta \mathrm{V}=$ systolic pulmonary arterial storage volume (ml.)

$\Delta \mathbf{P}=$ pulse pressure of lumped pulmonary arterial distending pressure $\left(\mathrm{cm} . \mathrm{H}_{2} \mathrm{O}\right)$

$\mathrm{Vo}=$ mean pulmonary arterial volume (ml.).

The pulmonary arterial pulse wave velocity could also be calculated from the BramwellHill equation:

$$
\mathrm{Co}=\sqrt{\frac{\mathrm{Vo}}{\mathrm{C}}}
$$

where $\mathrm{Co}=$ PA pulse wave velocity $(\mathrm{cm} . / \mathrm{sec}$.)

$$
\text { Vo }=\text { PA volume (ml.: from stroke }
$$
volume and ether time)

$\mathrm{C}=\mathrm{PA}$ compliance $\left(\mathrm{ml} . / \mathrm{cm} . \mathrm{H}_{2} \mathrm{O}\right)$

but from equation (i)

$$
\frac{\mathrm{V}}{\mathrm{C}}=\frac{\mathrm{IOO}}{\mathrm{D}}
$$


where $\mathrm{D}=$ lumped PA distensibility (\% vol. change $/ \mathrm{cm} . \mathrm{H}_{2} \mathrm{O}$ )

$$
\text { so } \quad \mathrm{Co}=\sqrt{\frac{\mathrm{IO0}}{\mathrm{D}}}
$$

Fourier analysis of pressure and flow data was also undertaken in order to examine the pulmonary arterial input impedance changes imposed by hypoxia and serotonin.

Fig. 4 shows the results of our compliance studies in 13 dogs. We found that there was a hyperbolic relation between the rising pulmonary arterial resistance and the falling pulmonary arterial compliance induced by graduated changes in hypoxia and serotonin infusion. Fig. 5 next shows that the pulmonary arterial time constant $(t)$ remained uniform over a very wide range of pulmonary arterial pressure induced by either hypoxia or serotonin infusion. Only above a mean pulmonary artery pressure of $40 \mathrm{~mm}$. $\mathrm{Hg}$ did the PA time constant begin to increase. The time constant (t) was calculated from the product of pulmonary arterial compliance (C) and pulmonary arterial resistance $(R)$. This finding gave a satisfactory explanation for the maintained pulsatility of lung capillary blood flow in conditions of moderate pulmonary arterial hypertension, for as precapillary resistance increased, tending to attenuate lung capillary flow pulsatility, its effect was counteracted by the decreased compliance of the pulmonary arterial system, tending to increase transmission of pulsatility to the capillaries. The effects of the decreasing distensibility of the pulmonary arterial system, produced by increasing degrees of pulmonary hypertension, had a natural consequence of increasing the pulmonary arterial pulse wave velocity. The relationship between the mean pulmonary arterial pressure and pulse wave velocity is shown in Fig. 6. Full details of this study have been published (Reuben et al., 1970a).

The pulmonary arterial input impedance calculations which we obtained from our instantaneous records of pulmonary arterial pressure and pulmonary capillary blood flow yielded fresh information about the site of resistance to blood flow in the arterial system of the lungs. We also found that the pulmonary arterial input impedance spectrum was independent of heart rate, which implies that the pulmonary arterial system behaves moderately linearly, for in a linear system an alteration in the harmonics of pressure by increased heart rate would be accompanied by identical changes in the harmonics of flow so that the ratio of the two, namely the impedance modulus, would still lie on the control slope but at a new point appropriate to the higher

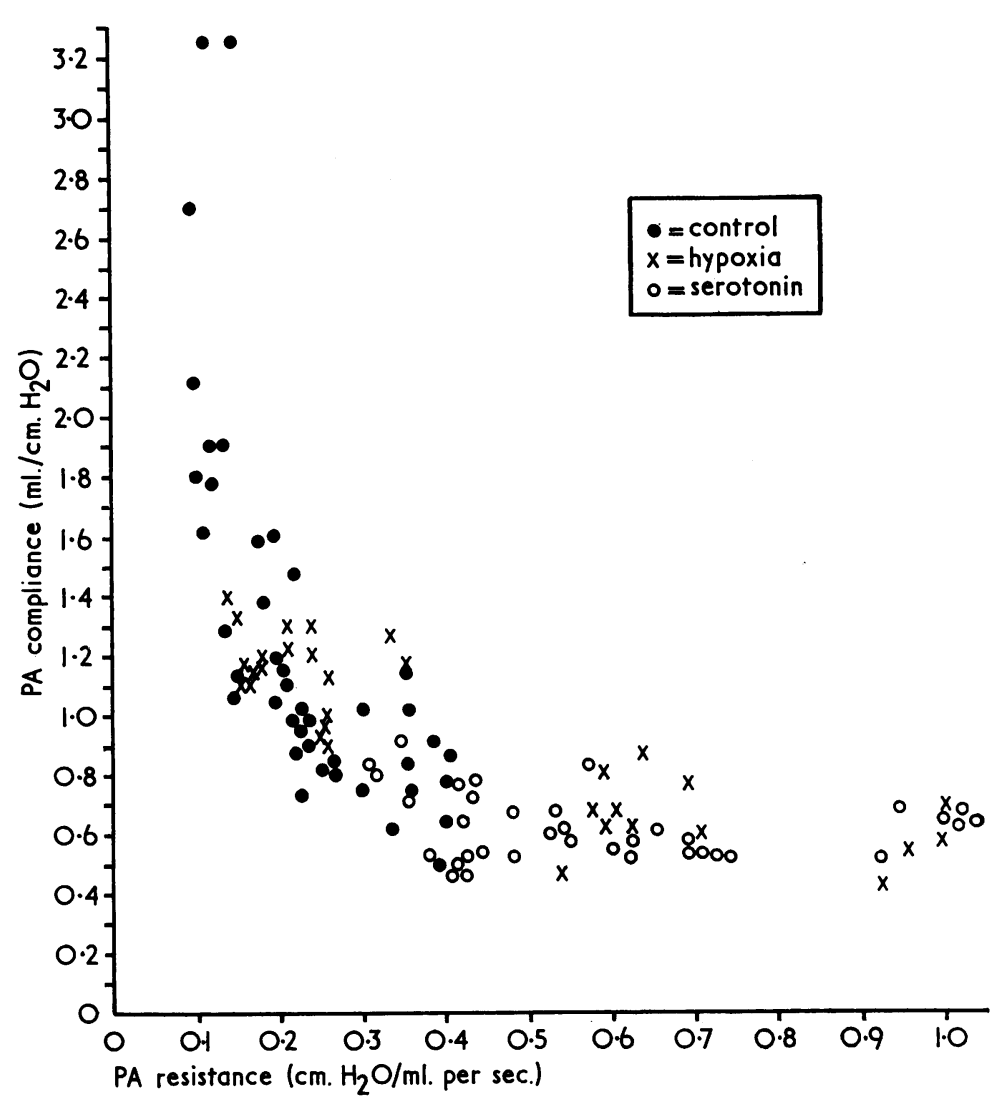

FIG. 4 The relationship between pulmonary arterial resistance and the corresponding value of compliance in dogs. The graph combines data from control $(\bullet)$, hypoxia $(\times)$, and serotonin (O) studies.

fundamental frequency imposed by the change in heart rate.

The pulmonary arterial input impedance spectrum during air breathing showed a first minimum value of 100 dyn.sec.cm. ${ }^{-5}$ occurring at 3.52 cycles per second (c.p.s.). A first maximum of 214 dyn.sec.cm. ${ }^{-5}$ took place at $9 \cdot 0$ c.p.s., followed by a second minimum at 13.0 c.p.s. The impedance phase angles were negative at low frequencies, indicating flow leading pressure, but they became positive above 3.0 c.p.s. The reflection coefficient during this period was calculated to be $0.3 \mathrm{I}$ and the average distance from the pulmonary valve to the reflecting site was $13.5 \mathrm{~cm}$.

Hypoxic ventilation caused a rise in the input resistance from 540 to 680 dyn.sec.cm. ${ }^{-5}$ and a shift in the frequency of the first impedance minimum to $5 \cdot 60$ c.p.s. The reflection coefficient for these data was calculated to be 0.49 , while the distance to the reflecting site 


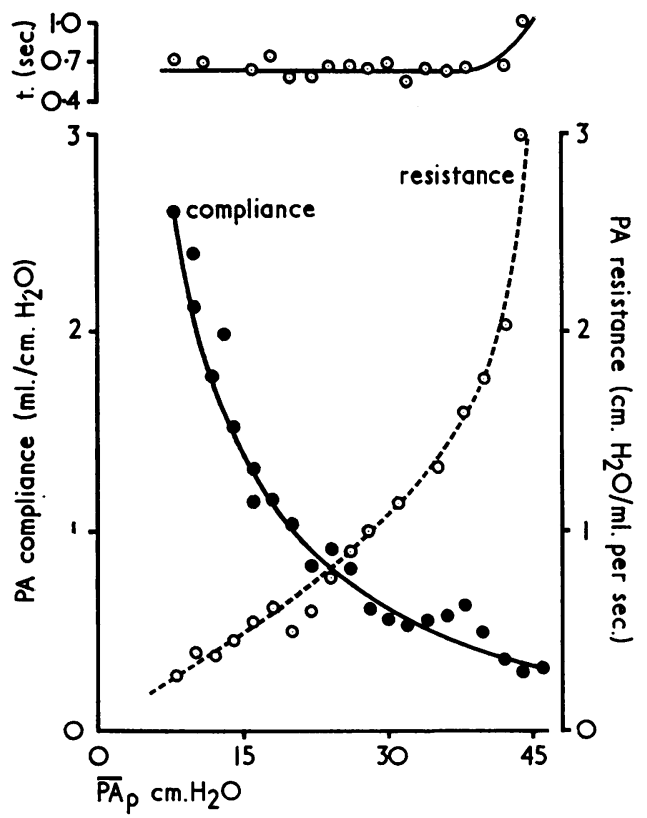

FIG. 5 The relationship between pulmonary arterial compliance $(C)$, pulmonary arterial resistance $(R)$, mean pulmonary artery pressure, and pulmonary arterial time constant $(t)$ obtained from the product of $(C \times R)$ in dogs. Data from control hypoxia and serotonin studies were combined to obtain these relationships.

fell to $10.2 \mathrm{~cm}$. Serotonin infusion caused an even greater rise in the input resistance to 850 dyn.sec.cm. ${ }^{-5}$ and a bigger shift of the first impedance minimum to 6.60 c.p.s. The reflection coefficient was calculated to be 0.53 and the distance to the reflecting site to be $12 \cdot 2 \mathrm{~cm}$.

The most dramatic change in the pulmonary arterial input impedance spectrum was produced by sympathetic nerve stimulation undertaken in two dogs. The input impedance rose to 780 dyn.sec.cm. ${ }^{-5}$ and the first impedance minimum shifted to 7.5 c.p.s., with a consequent large fall in the distance to the reflecting site to $8.7 \mathrm{~cm}$. and a rise in the reflection coefficient to 0.56 . The results of these studies have been published (Reuben et al., I970b).

These results indicate that acute pulmonary hypertension causes marked alterations in the distal pulmonary arterial bed characterized by an increased vascular resistance. The increase in wave reflection, and also the shift proximally of the site of wave reflection towards the pulmonary valve, indicate that the main site of resistance to blood flow in the pulmonary

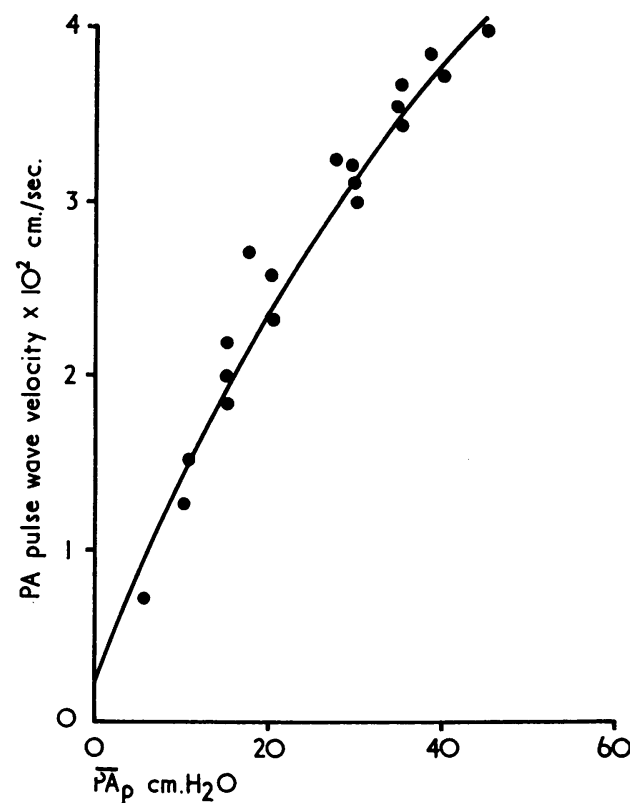

FIG. 6 The relationship between pulmonary arterial pulse wave velocity and mean pulmonary arterial input pressure $(\overline{P A} p)$. The pulse wave velocity was calculated from the compliance data shown in Fig. 4 using the Bramwell-Hill equation.

arterial system is capable of moving several $\mathrm{cm}$. proximally into the larger vessels of the pulmonary arterial system. This implies that hypoxia, serotonin, and sympathetic nerve action all have effects on the larger vessels of the pulmonary arterial system as well as at the more conventional resistance site in the arterioles.

Previous studies by Ingram et al. (1968) on isolated lobes of the lung in situ, with their nerve supply intact, had already shown that during sympathetic stimulation the larger pulmonary arteries became stiffer, whereas the calculated pulmonary arterial resistance increased only slightly. In contrast noradrenaline infusion produced less stiffness change in the larger pulmonary arteries but greater effects on the arterioles. Further studies (Ingram, Szidon, and Fishman, 1970) have shown that hypothalamic stimulation, acting via the sympathetic outflow, showed a predominant action upon the media of the larger pulmonary arteries, where the sympathetic nerve endings are located. These findings by Fishman's group have direct relevance to our pulmonary artery compliance studies for they show the mechanisms whereby the pulmonary arterial compliance is regulated in 


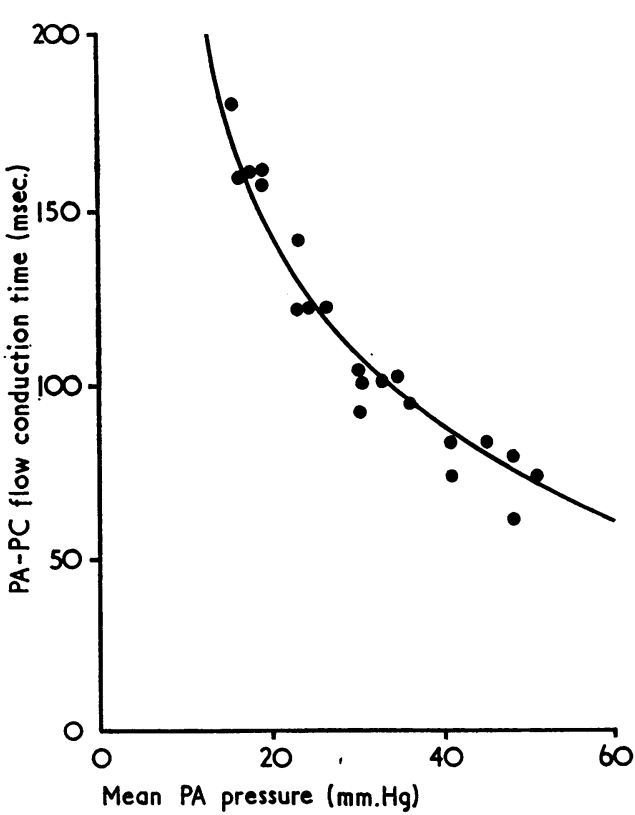

FIG. 7 The relationship between pulmonary arterial conduction time $(C t)$ and mean pulmonary artery pressure $(P A p)$ in man.

pulmonary hypertension and that the decreasing compliance is not a purely passive effect due to vascular distension.

The present consensus of opinion regarding the actions of chemical agents and reflexes upon the pulmonary circulation strongly suggests that hypoxia, pressor amines, and to a lesser extent hypercapnoea produce direct local vasocontrictor effects upon the arterioles and venules of the lung, but in addition reflex responses are excited which increase the stiffness of the more proximal pulmonary arteries. The problem now facing investigators is to design studies which will allow the relative importance of reflex and local action to be assessed in their relationship to one another within the pulmonary circulation.

The increased pulse wave velocity and pulmonary artery flow conduction time which were found by Reuben et al. (1970a), and which were associated with decreased pulmonary arterial compliance as the pulmonary artery pressure rose in animals, have excited Reuben to explore the possibility of using the conduction time as a means of calculating mean pulmonary artery pressure from the $\mathrm{N}_{2} \mathrm{O}$ uptake curve in patients in the body plethysmograph. He therefore re-examined Karatzas and Lee's data (1969) obtained from patients with heart disease in whom the pulmonary arterial pressure had been measured by cardiac catheterization simultaneously with $\mathrm{N}_{2} \mathrm{O}$ uptake in the body plethysmograph. $\mathrm{He}$ then correlated the pulmonary artery flow conduction time $(\mathrm{Ct})$ against mean pulmonary artery pressure. He found a curvilinear relationship between the two and was able to derive the mean pulmonary arterial pressure with great accuracy from measurements of the flow conduction time using nitrous oxide (Reuben, 1970). Reuben showed that the relationship between flow conduction time and mean pulmonary artery pressure conformed to the following equation:

$$
\mathrm{Ct}=31 \cdot 0+\frac{\text { 1910 }}{\overline{\mathrm{PA}} \mathrm{p}-2 \cdot 2}
$$

Role of the pulmonary venous system in regulating blood flow in the lung capillaries

Having briefly reviewed the regulation of the dynamics of blood flow into the lung capillary system by the pulmonary arteries, and how the alveolar capillary systems themselves contribute to regulating pulmonary vascular pressure and flow distribution in the lungs, it now remains necessary to examine the role of the pulmonary venous system.

Under normal conditions the $\mathrm{N}_{2} \mathrm{O}$ uptake curve, representing pulmonary capillary blood flow, virtually resembles the profile of the pulmonary arterial pressure curve though it is separated from it in time. Blood flow through the lung capillaries is therefore largely uninfluenced by pressure events occurring in the venous system of the lungs, which implies that pressure events from the left atrium are damped out from reaching the capillaries because of the anatomical characteristics of the pulmonary venous system. Part of the explanation for this may be inferred from work on the isolated rabbit lung by Caro and Saffman (1965). These workers showed that the pulmonary venous system has an extremely non-linear compliance, being highly distensible at low venous pressures but rapidly becoming indistensible as the venous pressure rises. Harris, Heath, and Apostolopoulos (1965) had examined the distensibility of the pulmonary arterial trunk in man and had found that it was considerably more distensible than the aorta. They used circumferential strips of these vessels, obtained at necropsy, and plotted length tension curves from them, using a travelling microscope after setting up the material in a tension balance. They did not extend their work to the pulmonary veins. I have carried out limited studies, using their technique on the pulmonary 
venous trunk in necropsy material and have compared it with the pulmonary arterial findings of Harris. My findings were identical with those of Harris and his associates with regard to the pulmonary artery. However, the pulmonary vein, though initially highly distensible, became virtually indistensible when its tension exceeded 2.5 dynes $/ \mathrm{mm}^{2}$ In terms of pressure related to vessel dimension, this means that at pressures exceeding $10-15 \mathrm{~mm}$. $\mathrm{Hg}$ a state of affairs will be reached where the pulmonary veins attain the limits of their distensibility. Pressure events occurring in the left atrium are then likely to be transferred retrograde to the venular end of the capillary system, having been damped out previously, and might be expected to impede lung capillary outflow in a manner which could be predicted from the shape of the left atrial pressure curve, provided that the arterial inflow profile was normal. In order to test the hypothesis that episodic changes in pulmonary venous impedance during the cardiac cycle might affect the pulsatility of lung capillary blood flow I have found it convenient to examine a somewhat contrived situation where these events are taking place in virtual isolation. This happens in patients with complete heart block in whom the ventricular rate is both fixed and slow and atrial contraction takes place at a faster rate, which is also randomly related to ventricular events (Gillespie $e t$ al., 1967). In this way episodic changes in pulmonary venous impedance were produced by the contractions of the left atrium, which varied randomly throughout the ventricular cycle. During ventricular diastole the effects of pulmonary venous pressure fluctuation transmitted from the left atrium could be studied by searching for alterations in the normal lung capillary blood flow profile in relation to the $P$ wave of the electrocardiogram. In patients with congenital complete heart block, where the left atrial pressure was normal, the left atrial pressure events produced very small change in lung capillary blood flow. However, when the left atrial pressure was high, as in acquired complete block with heart failure, an entirely different capillary blood flow profile was obtained. Under these circumstances, with high left atrial pressures, cannon waves from the left atrium were transmitted retrograde through the pulmonary venous system to the capillaries and at times could be picked up by pressure monitoring in the pulmonary artery. Under these circumstances $\mathrm{N}_{2} \mathrm{O}$ uptake from the lung capillaries virtually ceased at the times when episodic increases in venous outflow impedance were produced by the cannon wave (Lee, 1969a).
More recently I have been studying the effect of isolated mitral incompetence upon lung capillary blood flow pulsatility. In patients where the main pulmonary artery pressure is normal at rest but who have severe mitral incompetence resulting in giant left atrial $\mathrm{V}$ waves, the left atrial $\mathrm{V}$ wave may approach some $40 \mathrm{~mm}$. $\mathrm{Hg}$ and be conducted retrograde to the pulmonary arterial system. During these periods $\mathrm{N}_{2} \mathrm{O}$ uptake from the lungs virtually ceases, and indeed lung capillary blood flow in mitral incompetence under these circumstances virtually depends on a long diastolic interval for resumption of blood flow from the pulmonary artery to the pulmonary veins (Lee 1969b). Normal pulsatile lung capillary blood flow events may be reinstated by replacement of the leaking mitral valve by means of a Starr-Edwards ball valve. These $\mathrm{N}_{2} \mathrm{O}$ studies provide an explanation for surprising dyspnoea sometimes encountered in patients with pure mitral incompetence and normal pulmonary arterial pressures at rest, who become intensely breathless on exertion if this is associated with a rapid increase in heart rate. Heart rate control with digitalis and propranolol is often dramatically beneficial in such cases, because of the increased diastolic flow time through the lung capillaries produced by good heart rate control.

These clinical observations allow only intuitive deductions to be made about the role of the pulmonary veins and left atrium in modifying the transmission of pressure and flow through the lung vasculature. Precise physiological study of the role of the pulmonary veins in regulating lung capillary flow are virtually non-existent at the time of preparing this review. However, Caro, Bergel, and Seed (1967) have examined the transmission of pressure waves through the pulmonary vascular bed. They measured pressure in the lobar pulmonary arteries, veins, and left atria of anaesthetized open-chested dogs. Observations were made before and after snaring the lobar vessels and before and after infusions of dextran to raise the mean pressure. Forward and backward transmission (output/input pressure) and transmission ratios (forward/ backward transmission) were calculated from the pressure waves submitted to Fourier analysis. At low frequencies the vascular bed was non-symmetrical, forward transmission (arteries to veins) being several times greater than backward transmission (veins to arteries) at the same pressure. These findings would suggest that at low pulmonary vein pressure the PA pressure and flow pulse will be damped out in its passage through the venous system so that it becomes more uniform. We 
have been able to confirm this in our laboratory, measuring pulmonary vein inflow to the left atrium using an electromagnetic flowmeter. Our findings confirm the findings of Kinnen and Stankus (1968), who showed that the velocity of blood flow in the pulmonary veins entering the left atrium is largely uniform but also influenced by the pressure events taking place locally in the left atrium. However, the work of Caro et al. (1967) also suggests that if the left atrium and pulmonary venous pressure was high, so that the veins became non-compliant, then arterial pulsations of pressure and flow would be transmitted across the capillary system into the veins so that a more 'arterial' venous flow pulse would be observed. It is interesting that Morkin et al. (1965) had described such pulsatility of venous flow by direct measurements in dogs and had concluded that this venous pulsatility was transmitted from the right heart through the arterial and capillary systems to dominate venous flow. It was relevant that their measurements were conducted at moderately high pulmonary venous pressures (Io mm. $\mathrm{Hg}$ ). Controversy soon arose about the discrepancies in flow velocity profile found by various workers in the pulmonary veins. Our current studies in animals indicate that the mean level of pressure in the left atrium and pulmonary veins dominates the behaviour of venous outflow impedance to blood flow from the lung capillaries. Thus, when left atrial pressure is low, pulmonary venous inflow to the left atrium is moderately uniform, being momentarily impeded by left atrial systole. However, if the left atrial pressure is elevated by inflating a water-filled balloon in the left atrium of closed-chested dogs the pulmonary venous flow to the left atrium becomes highly pulsatile and resembles a damped lung capillary blood flow pulsation, delayed in time from capillary events. Considerable further work is needed before we will have acquired as precise a knowledge of venous influences upon lung capillary blood flow as we now possess with regard to arterial function in the lungs.

\section{References}

Banister, J., and Torrance, R. W. (1960). The effects of the tracheal pressure upon flow: pressure relations in the vascular bed of isolated lungs. Quarterly fournal of Experimental Physiology and Cognate Medical Sciences, 45, 352.

Bates, D. V., Varvis, C. J., Donevan, R. E., and Christie, R. V. (1960). Variations in the pulmonary capillary blood volume and membrane diffusion component in health and disease. Fournal of Clinical Investigation, 39, $140 \mathrm{O}$.

Bosman, A. R., Honour, A. J., Lee, G. de J., Marshall, R., and Stott, F. D. (1964). A method for measuring instantaneous pulmonary capillary bloodflow and right ventricular stroke volume in man. Clinical Science, 26, 247.

Caro, C. G., Bergel, D. H., and Seed, W. A. (1967). Forward and backward transmission of pressure waves in the pulmonary vascular bed of the dog. Circulation Research, 20, 185.

- , and Saffman, P. S. (I965). Extensibility of blood vessels in isolated rabbit lungs. Fournal of Physiology, I78, 193.

Cudkowicz, L. (1968). The Human Bronchial Circulation in Health and Disease. Williams and Wilkins, Baltimore.

Feisal, K. A., Soni, J., and DuBois, A. B. (1962). Pulmonary arterial circulation time, pulmonary arterial blood volume, and the ratio of gas to tissue volume in the lungs of dogs. Fournal of Clinical Investigation, 4I, 390.

Gillespie, W. J., Greene, D. G., Karatzas, N. B., and Lee, G. de J. (1967). Effect of atrial systole on right ventricular stroke output in complete heart block. British Medical fournal, $\mathbf{x}, 75$.

Glazier, J. B., Hughes, J. M. B., Maloney, J. E., Pain, M. C. F., and Webb, J. B. (1966). Decreasing alveolar size from apex to base in the upright lung. Lancet, 2, 203.

,$--\longrightarrow$, Rosenzweig, D. Y., and West, J. B. (I968). Effect of vascular pressures on pulmonary capillary morphology. Clinical Research, 16, 370.

Harris, P., Heath, D., and Apostolopoulos, A. (I965). Extensibility of the human pulmonary trunk. British Heart fournal, 27, 65I.

Heinemann, H. O., and Fishman, A. P. (1969). Non respiratory functions of mammalian lung. Physiological Reviews, 49, I.

Howell, J. B. L., Permutt, S., Proctor, D. F., and Riley, R. L. (196I). Effect of inflation of the lung on different parts of the pulmonary vascular bed. fournal of Applied Physiology, 16, 71.

Hughes, J. M. B., Glazier, J. B., Maloney, J. E., and West, J. B. (1968). Effect of lung volume on the distribution of pulmonary blood flow in man. Respiration Physiology, 4, 58.

Ingram, R. H., Jr., Szidon, J. P., and Fishman, A. P. (1970). Response of the main pulmonary artery of dogs to neuronally released versus blood-borne norepinephrine. Circulation Research, 26, 249.

,-- Skalak, R., and Fishman, A. P. (1968). Effects of sympathetic nerve stimulation on the pulmonary arterial tree of the isolated lobe perfused in situ. Circulation Research, 22, 801 .

Karatzas, N. B., and Lee, G. de J. (1966). The effect of pulsatile capillary blood flow on gas exchange within the lungs; a consideration of some hydrodynamic factors which affect this pulsatility in man. Bulletin de Physio-pathologie Respiratoire, 2, $52 \mathrm{I}$.

,-- (1969). Propagation of blood flow pulse in the normal human pulmonary arterial system. Analysis of the pulsatile capillary flow. Circulation Research, 25, 1 I.

,-- (1970). Instantaneous lung capillary blood flow in patients with heart disease. Cardiovascular Research, 4, 265 .

,-- , and Stott, F. D. (1967). A new electropneumatic flowmeter for the body plethysmograph. fournal of Applied Physiology, 23, 276.

Kinnen, E., and Stankus, A. J. (1968). Pulmonary venous blood flow. U.S.A.F. Report No. SAMTR-68-22.

Lee, G. de J. (I969a). Blood flow in the lungs. In Modern Trends in Cardiology, vol. 2, p. 197. Ed. by A. M. Jones. Butterworths, London.

(1969b). Lung blood flow studies in man using the whole body-plethysmograph: technical aspects and applications. Progress in Respiration Research, 4, 140. 
Lee, G. de J., and DuBois, A. B. (1955). Pulmonary capillary blood flow in man. Fournal of Clinical Investigation, 34, 1380.

- Matthews, M. B., and Sharpey-Schafer, E. P. (1954). The effect of the Valsalva manoeuvre on the systemic and pulmonary arterial pressure in man. British Heart fournal, 16, $31 \mathrm{I}$.

Mead, J., Takishima, T., and Leith, D. (1967). Mechanical interdependance of distensible units in the lung. Federation Proceedings, 26, 551.

Menkes, H. A., Sera, K., Rogers, R. M., Hyde, R. W., Forster, R. E., II, and DuBois, A. B. (1970). Pulsatile uptake of $\mathrm{CO}$ in the human lung. Fournal of Clinical Investigation, 49, 335.

Milic-Emili, J., Henderson, J. A. M., Dolovich, M. B., Trop, D., and Kaneko, K. (1966). Regional distribution of inspired gas in the lung. fournal of Applied Physiology, 21, 749.

Morkin, E., Collins, J. A., Goldman, H. S., and Fishman, A. P. (1965). Pattern of blood flow in the pulmonary veins of the dog. Fournal of Applied Physiology, 20, 1118 .

, Levine, O. R., and Fishman, A. P. (1964). Pulmonary capillary flow pulse and the site of pulmonary vasocontriction in the dog. Circulation Research, 15, 146.

Permutt, S. (1965). Effect of interstitial pressure of the lung on pulmonary circulation. Medicina Thoracalis, 22, 118.

- Bromberger-Barmea, B., and Bane, H. N. (1962). Alveolar pressure, pulmonary venous pressure, and the vascular waterfall. Medicina Thoracalis, 19, 239.

Reuben, S. R. (1970). Wave transmission in the pulmonary arterial system in disease in man. Circulation Research, 27, 523.
Gersh, B. J., Swadling, J. P., and Lee, G. de J. (I970a). Measurement of pulmonary arterial distensibility in the dog. Cardiovascular Research 4, 47 ?.

—, Swadling, J. P., Gersh, B. J., and Lee, G. de J. (1970b). Impedance and transmission properties of the pulmonary arterial system. Cardiovascular Research, 5, I.

Rosenzweig, D. Y., Hughes, J. M. B., and Glazier, J. B. (1970). Effects of transpulmonary and vascular pressures on pulmonary blood volume in isolated lung. Fournal of Applied Physiology, 28, 553.

Roughton, F. J. W., and Forster, R. E. (1957). Relative importance of diffusion and chemical reaction rates in determining rate of exchange of gases in the human lung, with special reference to true diffusing capacity of pulmonary membrane and volume of blood in the lung capillaries. Fournal of Applied Physiology, 11, 290.

Sackner, M. A., Will, D. H., and DuBois, A. B. (1966). The site of pulmonary vasomotor activity during hypoxia or serotonin infusion. Fournal of Clinical Investigation, 45, I1 2.

Wasserman, K., Butler, J., and Van Kessel, A. (1966) Factors affecting the pulmonary capillary flow pulse in man. Fournal of Applied Physiology, 21, 890 .

Weibel, E. R. (1963). Morphometry of the Human Lung. Springer, Berlin.

West, J. B., and Dollery, C. T. (1960). Distribution of blood flow and ventilation-perfusion ratio in the lung, measured with radioactive carbon dioxide. fournal of Applied Physiology, 15, 405.

, - and Naimark, A. (I964). Distribution of blood flow in isolated lung: relation to vascular and alveolar pressures. Fournal of Applied Physiology, 19, 713. 